\title{
Improvement of magnetorheological greases with superparamagnetic nanoparticles
}

\author{
Norzilawati Mohamad ${ }^{1}$, Ubaidillah", ${ }^{2,3}$, Saiful Amri Mazlan ${ }^{1, *}$,Seung-Bok Choi ${ }^{4}$ and Naim \\ Abdul Halim ${ }^{1}$ \\ ${ }^{1}$ Malaysia Japan International Institute of Technology, Universiti Teknologi Malaysia (UTM), Jalan \\ Sultan Yahya Petra, 54100 Kuala Lumpur, Malaysia \\ ${ }^{2}$ Mechanical Engineering Department, Faculty of Engineering, Universitas Sebelas Maret (UNS), Jl. \\ Ir. Sutami 36A, Kentingan Surakarta, 57126, Central Java, Indonesia \\ ${ }^{3}$ National Center for Sustainable Transportation Technology (NCSTT), Indonesia \\ ${ }^{4}$ Mechanical Engineering Department, Inha University, Incheon, Republic of Korea
}

\begin{abstract}
Magnetorheological greases (MRGs) is a viscoelastic suspension comprised of carbonyl iron (CI) particles in a thixotropic medium; grease, which able to solve the instability problem that occurs in oil medium like MRFs. The primary purpose introduction of superparamagnetic nanoparticles $\gamma-\mathrm{Fe}_{2} \mathrm{O}_{3}$ in MRGs suspension is to investigate the effect of additives toward MRGs characteristics. Two types of MRGs suspension with and without nanoparticles $\gamma-\mathrm{Fe}_{2} \mathrm{O}_{3}$ were prepared in the same CI particles concentration for comparison purpose. It was observed that MRGs with incorporated of $1 \mathrm{wt} \%$ nanoparticles $\gamma$ $\mathrm{Fe}_{2} \mathrm{O}_{3}$ showed an increment regarding magnetic properties. In the meantime, the off-state viscosity of MRGs containing nanoparticles $\gamma$ $\mathrm{Fe}_{2} \mathrm{O}_{3}$ was reduced, however, increased during on-state condition. This fact indicates that the interspaces between CI particles are filled by the nanoparticles $\gamma-\mathrm{Fe}_{2} \mathrm{O}_{3}$. Therefore, the introduction of the nanoparticles indirectly improved the MRGs properties.
\end{abstract}

\section{Introduction}

Magnetorheological (MR) materials have been attracting researcher intention in last six decades. This material comprises of non-magnetic viscoelastic materials such as fluids, greases, plasticine, and elastomer, filled with micron-sized soft magnetic materials [1-3]. The intrinsic properties of this smart material are responsive to the occurrence of magnetic fields and reversible to the initial state rapidly in the absence of magnetic fields [4]. Among several types of MR materials, MR fluids (MRFs) have been most attractive due to their broad application. However, according to Chand et al. [5], MRFs has been facing some problems such as settling rate and fluid leaking. These problems can be overcome by introducing thixotropic materials such as greases as the matrix carrier [6].

There is a very limited number of studies related to MR greases (MRGs) have been reported. Park et al. [7] stated that there are no sedimentation drawbacks in MRGs due to the greases property which able to suspend the particles against gravity. As has been reported by Mohamad et al. [8] MRGs have lower the off-state viscosity and yield stress

*Corresponding author: ubaidillah_ft@staff.uns.ac.id ; amri.kl@utm.my 
compared to the MRFs. Meanwhile, Kim et al. [9] stated that by adding an additive like kerosene oil in the grease medium have reduced the viscosity of MRGs and at the same time lower the yield stress.

Therefore, in this study, superparamagnetic nanoparticles $\gamma-\mathrm{Fe}_{2} \mathrm{O}_{3}$ was introduced to the MRGs as an additive. The primary goal of this study is to investigate the effect of superparamagnetic nanoparticles $\gamma-\mathrm{Fe}_{2} \mathrm{O}_{3}$ towards MRGs characteristics. The preparation of MRGs with nanoparticles (MRGN) was purely mechanical mixing. The amount of nanoparticles $\gamma-\mathrm{Fe}_{2} \mathrm{O}_{3}$ incorporated in MRGs was about $1 \mathrm{wt} \%$. Some tests were undertaken to evaluate their morphology, magnetization saturation, and rheological properties.

\section{Materials and testing}

Soft magnetic carbonyl iron (CI) particles (standard OM grade, BASF, Germany) with an average diameter of $6 \mu \mathrm{m}$ and density $7.86 \mathrm{~g} \mathrm{ml}^{-1}$ were used as received without any further chemical treatment. Commercial grease (NPC Highrex HD-3 Grease, Nippon Koyu Ltd, Japan) with NLGI 3 was selected as suspending medium. The nanoparticles of $\gamma-\mathrm{Fe}_{2} \mathrm{O}_{3}$ were synthesized thru co-precipitation method described by Leong et al. [10]. Two types of MRGs were prepared which were CI particles based MRGs with and without nanoparticles $\gamma-\mathrm{Fe}_{2} \mathrm{O}_{3}$ as an additive. Firstly, CI particles (70 wt $\left.\%\right)$ and nanoparticles $\gamma-\mathrm{Fe}_{2} \mathrm{O}_{3}(1 \mathrm{wt} \%)$ were dispersed in ethanol. The suspension was stirred using mechanical stirrer and mixed for two hours to produce a more homogenized suspension. The particles size distribution of MRGN were determined using environmental scanning electron microscope (ESEM). Meanwhile, the magnetic properties and magneto-induced viscosity of MRGs and MRGN were examined using vibrating sample magnetometer (VSM) and rheometer, respectively. All the samples characterizations were conducted at room temperature $\left(25^{\circ} \mathrm{C}\right)$.

\section{Result and discussion}

Figure 1 depicts a morphological photograph of MRGN in which the nanoparticles can be seen dispersed within the micron-sized particles. However, some micron-sized particles are still aggregated due to the carrier phase.

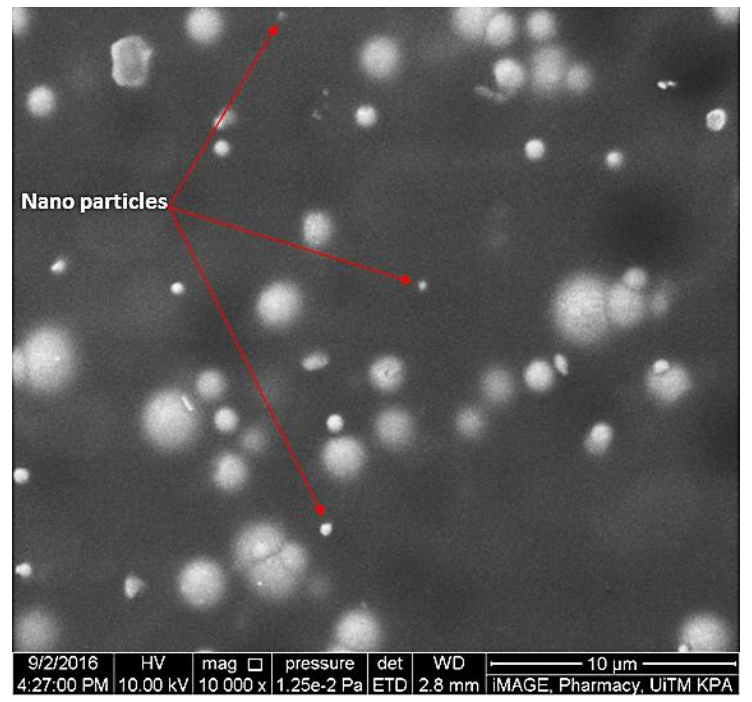

Fig. 1. Distribution of CI particles and nanoparticles $\gamma-\mathrm{Fe}_{2} \mathrm{O}_{3}$ in suspending medium of grease. 
The magnetization saturation (Figure 2) was measured through vibrating sample magnetometer (VSM) showing that there is a significant improvement of magnetization saturation. The MRGs has magnetization saturation of about $130 \mathrm{emu} / \mathrm{g}$ while the MRGN has a much higher value of about $201 \mathrm{emu} / \mathrm{g}$. Also, the hysteresis loop of MRGN is very narrow with a small coercivity compared to MRGs which indicates that nanoparticles $\gamma$ $\mathrm{Fe}_{2} \mathrm{O}_{3}$ also behave as soft materials that important for reversibility of MR performance [11]. It was proven that the superparamagnetic nanoparticles had improved the magnetic properties.

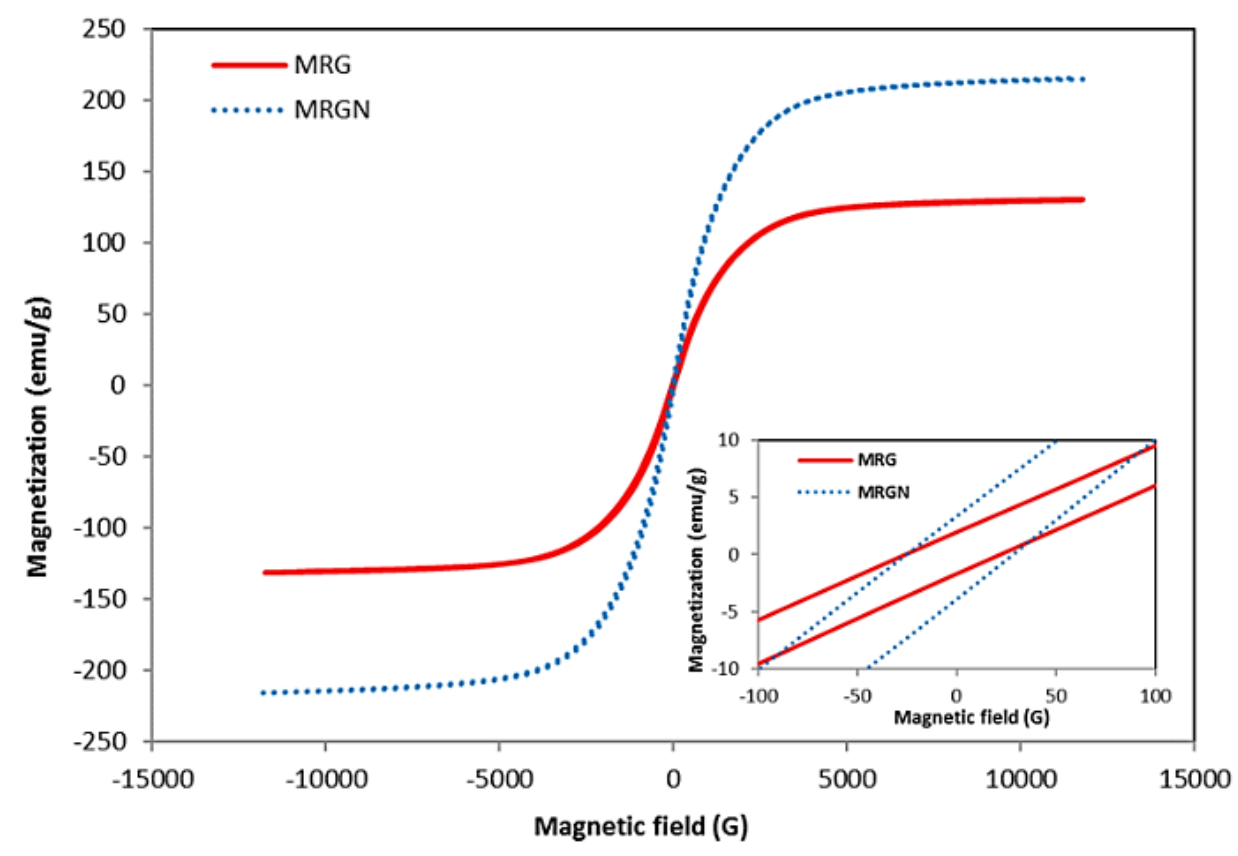

Fig. 2. Comparison of magnetic properties between MRGs and MRGN.

Moreover, rotational rheometer was used to evaluate the magneto-induced viscosity from 0 to 2000 1/s shear rate. As seen in Figure 3, the magneto-induced viscosity of MRGN and MRGs demonstrate a typical shear-thinning behavior. The introduction of nanoparticles $\gamma-\mathrm{Fe}_{2} \mathrm{O}_{3}$ has lowered the off-state viscosity of MRGs. However, during on-state which by applying current $4 \mathrm{~A}$ or equivalent to $658 \mathrm{mT}$, the viscosity of MRGN is higher than that of MRGs. As expected, this phenomenon is due to the nanoparticles $\gamma-\mathrm{Fe}_{2} \mathrm{O}_{3}$ had attached and filled the interspaces between the micron-sized particles when the magnetic field is applied $[12,13]$. Thus, the formation of regular and compact chains structure in the MRGN is attained, indirectly, enhance the MR performance. 


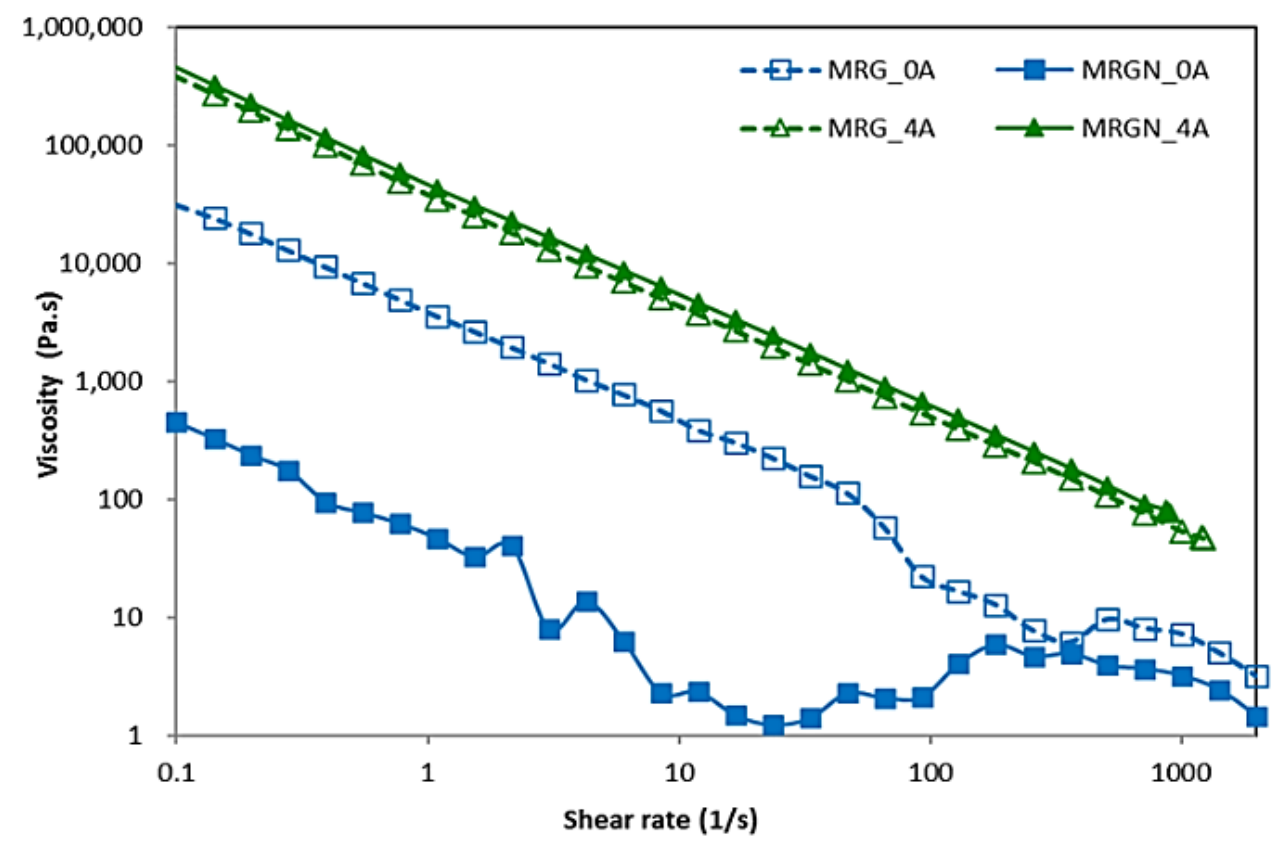

Fig. 3. Comparison of magneto-induced viscosity between MRGs and MRGN.

\section{Conclusion}

In this work, the influence of superparamagnetic nanoparticles in the MRGs have been investigated. Two types of MRGs were prepared with and without incorporation of nanoparticles $\gamma-\mathrm{Fe}_{2} \mathrm{O}_{3}$ for comparison purposes. It has been observed that MRGN showed a significant increment in magnetization saturation compared to the MRGs. It has also been observed that the MRGs incorporated of superparamagnetic nanoparticles $\gamma-\mathrm{Fe}_{2} \mathrm{O}_{3}$ has reduced the off-state viscosity but slightly increased the on-state viscosity. This fact is due to the nanoparticles $\gamma-\mathrm{Fe}_{2} \mathrm{O}_{3}$ has filled in the voids between micron-size particles in grease medium. As a conclusion, the introduction of superparamagnetic nanoparticles $\gamma-\mathrm{Fe}_{2} \mathrm{O}_{3}$ has been proven for improving the intrinsic properties of the MRGs.

This research was financially supported by: SHERA Project through Prime Award: AID-497-A-1600004, USAID as well as the Universiti Teknologi Malaysia under Tier 1 Grant (Vote No: 13H55)

\section{References}

1. K. Hairuddin, Ubaidillah, S.A. Mazlan, H. Zamzuri, and N.M. Nor, Appl. Mech. Mater. 660, 763 (2014).

2. N. Rosenfeld, N.M. Wereley, R. Radakrishnan, and T.S. Sudarshan, Int. J. Mod. Phys. B 16, 2392 (2002).

3. A.Z. Pokaad, K. Hudha, M.Z.B.M. Nasir, and N.A. Ubaidillah, Int. J. Struct. Eng. 2, 164 (2011).

4. B. Ichwan, S.A. Mazlan, F. Imaduddin, Ubaidillah, T. Koga, and M.H. Idris, Smart Mater. Struct. 25, 37001 (2016). 
5. M. Chand, A. Shankar, K. Jain, and R.P. Pant, RSC 4, 53960 (2014).

6. Ubaidillah, J. Sutrisno, A. Purwanto, and S.A. Mazlan, Adv. Eng. Mater. 17, 563 (2015).

7. B.O. Park, B.J. Park, M.J. Hato, and H.J. Choi, Colloid Polym. Sci. 289, 381 (2011).

8. N. Mohamad, S.A. Mazlan, Ubaidillah, S. Choi, and M.F.M. Nordin, Smart Mater. Struct. 25, 95043 (2016).

9. J.E. Kim, J.-D. Ko, Y.D. Liu, I.G. Kim, and H.J. Choi, IEEE Trans. Magn. 48, 3442 (2012).

10. S.A.N. Leong, P. Mohd Samin, A. Idris, S.A. Mazlan, and A.H.A. Rahman, Smart Mater. Struct. 25, 25025 (2016).

11. S.A.N. Leong, S.A. Mazlan, P.M. Samin, A. Idris, and Ubaidillah, AIP Conf. Proc. 1710, (2016).

12.N.M. Wereley, A. Chaudhuri, J.-H. Yoo, S. John, S. Kotha, A. Suggs, R. Radhakrishnan, B.J. Love, and T.S. Sudarshan, J. Intell. Mater. Syst. Struct. 17, 393 (2006).

13. E.F. Burguera, B.J. Love, R. Sahul, G. Ngatu, and N.M. Wereley, J. Intell. Mater. Syst. Struct. 19, 1361 (2008). 